\title{
BIOGENIC AMINES IN RETT SYNDROME
}

Significant reductions in CSF metabolites of norepinephrine, dopamine, and serotonin are reported in 32 female patients with suspected Rett syndrome from the Baylor College of Medicine, Houston, Texas. CSF biopterin, an essential cofactor that may limit the synthesis of biogenic amines, was elevated in patients compared with controls. Diet, drugs, and nutritional status, that may affect monoamine metabolites, were thought to be unlikely explanations for these biochemical changes. (Zoghbi HY et al. Cerebrospinal fluid biogenic amines and biopterin in Rett syndrome. Ann Neurol Jan $1989 ; \underline{25}: 56-60$ ).

COMMENT. Abnormal biochemical findings reported in Rett syndrome, including hyperammonemia, have not been substantiated. If specific and unexplained by anticonvulsant drugs or diet, this report is the first to suggest a metabolic disorder underlying the stereotypic hand movements and other neurologic signs of Rett syndrome. Abnormal CSF biogenic amines are also reported in Parkinson's disease, Huntington's chorea, and LeschNyhan syndrome.

\section{MENKES DISEASE WITH 'RAGGED RED' FIBERS}

Subsarcolemmal aggregates of mitochondria ('ragged red' fibers) in skeletal muscle were found at autopsy in a 30-monthold male infant with Menkes kinky-hair disease reported from the New York Hospital-Cornell University Medical Center, New York. At birth, the infant had multiple depressed skull fractures and a cephalhematoma. At 5 days, he developed vertical nystagmus and staring episodes; at 2 months, generalized seizures; at 4 months, multifocal myoclonic twitches; and by 6 months, he had hypotonia, poor head control, and visual inattention. His height, weight, and head circumference were at the 3 rd percentile. His hair was sparse, poorly pigmented, and showed pili torti (twisted), monilethrix (beadlike) and trichorrhexic nodosa (fractured nodes) on microscopic examination. The plasma copper was $16 \mathrm{mcg} / 100 \mathrm{ml}$ (normal: approx $100 \mathrm{mcg} / 100 \mathrm{ml}$ ), and ceruloplasmin 12mg/dl (normal: 15-50mg/dl). He was treated with sodium valproate for seizures, and he had multiple episodes of vomiting, weight loss and dehydration, and respiratory infections. The immediate cause of death was bronchopneumonia. The brain weighed $500 \mathrm{~g}$ (normal: $1100 \mathrm{~g}$ ) and showed diffuse cerebral and cerebellar atrophy, with focal polymicrogyria. Electronmicroscopy demonstrated numerous mitochondria within Purkinje cell cytoplasm. This report, "the first to describe 'ragged red' fibers in Menkes disease," supports the concept that Menkes disease may be due in part to a mitochondrial enzyme deficiency. (Morgello $\mathrm{s}$ et al. Menkes kinky hair disease with 'ragged red' fibers. Dev Med Child Neurol Dec 1988;

COMMENT. Menkes kinky hair disease is an X-chromosome linked disorder of copper malabsorption characterized by low serum ceruloplasmin and copper levels, seizures, CNS degeneration, and pili torti (Menkes JH et al. Pediatrics

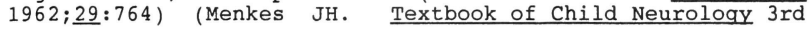


Edition 1985, Lea \& Febger, Philadelphia). Parenterally administered copper corrects the hepatic copper deficiency and restores serum copper and ceruloplasmin levels to normal but may not arrest the progressive cerebral degeneration. Nonetheless, Menkes advises initiation of copper therapy early since the clinical course of the disease is variable.

\section{NEURO-OPHTHALMOLOGY}

\section{TONIC UPGAZE IN INFANCY}

Tonic upward ocular deviation without seizure activity or neurologic disease is reported in 3 infants from the Pediatric and Neuro-ophthalmology Units, School of Medicine, University of California, San Francisco. The frequency and duration of the tonic upgaze episodes decreased with time but were exacerbated by fatigue or illness. Downward eye movements were normal. (Ahn JC et al. Tonic upgaze in infancy. A report of three cases. Arch Ophthalmol Jan 1989;107:57-58).

COMMENT. Transient tonic downward gaze in newborns is a fairly common phenomenon whereas tonic upgaze deviation is rarely reported, except with seizure activity, visual loss, or brain-stem disease. The pathophysiology of this transient variety of tonic upgaze is unknown but appears to differ from that of the downgaze type, being more persistent and exacerbated by fatigue or illness.

\section{OCULAR SIGNS OF CONGENITAL VARICELIAA}

Ocular abnormalities associated with congenital varicella are described in 3 children examined at the Hospital for Sick Children, Great Ormond Street, London, England. The syndrome followed a maternal varicella infection during the second trimester of pregnancy. Ocular findings included chorioretinitis, atrophy and hypoplasia of optic discs, cataract, and Horner's syndrome. Neurological abnormalities included bulbar palsy, hemiparesis, learning difficulties, and psychomotor retardation. The authors stress that the ocular lesions may be subtle and routine ophthalmological evaluation in infants with the congenital varicella syndrome is advisable. (Lambert SR, Taylor D et al. Ocular manifestations of the congenital varicella syndrome. Arch Ophthalmol Jan 1989;107: $52-56$ ).

COMMENT. Chorioretinal scarring occurs after intrauterine rubella, toxoplasmosis, syphilis, and less frequently with intrauterine herpes simplex and cytomegalovirus infections. Varicella chorioretinitis should be included in the differential diagnosis of congenital chorioretinal scarring. Optic nerve hypoplasia has not previously been reported in association with congenital varicella syndrome. 